\title{
ELEGANCE IN SCIENCE AND SCIENCE EDUCATION
}

\author{
Boris Aberšek \\ University of Maribor, Slovenia
}

Science is often thought of as a methodical but dull activity. However, the finest science, the breakthroughs most admired and respected by scientists themselves, are characterized by elegance. Thinking of elegance as grace, taste or refined luxury would be useless here. So what does elegance mean in the context of science? Economy is a considerable part of it, as is creativity. An elegant mathematical proof, an elegant theory, or an elegant experiment are all, that is, economical and imaginative, and sometimes breathtakingly simple, once explained.

When it comes to elegance, however, what do we mean? What does the word elegance truly designate? It comes from the Latin elegantia, "taste, propriety, or refinement." Elegance is usually a synonym for beauty and has acquired the additional connotations of unusual effectiveness and simplicity. The word is frequently used as a standard of tastefulness, particularly in the areas of visual design, decoration, the sciences and the aesthetics of mathematics.

Essential components of the concept of elegance include simplicity and consistency of design, focusing on the essential features of an object. In mathematics it can be used to describe the proof of a mathematical theorem, which exhibits mathematical elegance if it is surprisingly simple yet effective and constructive; similarly, a computer program or algorithm is elegant if it uses a small amount of code to great effect. On the other hand, in technology and engineering, a solution may be considered elegant if it uses a non-obvious method to produce a solution that is highly effective and simple. Elegance has been promoted as a new watchword to guide the next step within the current cycle of engineering and technological innovation. Elegance in engineering terms means achieving a reduction in technological complexity, thereby preserving an underlying organizational complexity. In short: Engineering elegance articulates complexity. An elegant solution may solve multiple problems at once, especially problems not thought to be interrelated. There's an old expression, "Don't work harder; work smarter." Old as it may be, this is one of the adages of New Purchasing: The answer to complexity does not have to be more complexity. Complexity does not have to mean confusion or latency. Problem solving will become quicker and more elegant. There will be simplified and elegant solutions that can do much of the heavy lifting for us (Glyn, 2010).

\section{Elegance in Science}

So let me try to explain what I mean by "elegance in science" by considering two different ways of solving a mathematical problem, a problem of no other use, of no profundity, and no importance. It is this (Glyn, 2010).

In considering the Pythagorean Theorem, we must acknowledge that how, when and where the theorem was first proved is not known; however, three centuries after Pythagoras, Euclid provided a proof that has remained the standard scientific proof. It involves a slightly complicated construction (see Figure 1) and the application of theorems about congruent triangles, and about the relation between the areas of the triangles and rectangles that are on the same base and between the same parallels--to use the traditional jargon. This proof is entirely convincing, but it mostly lacks the "wow" factor. However, there is a much simpler proof, the origin of which is obscure, although it was certainly known in the nineteenth century. It goes as follows: 


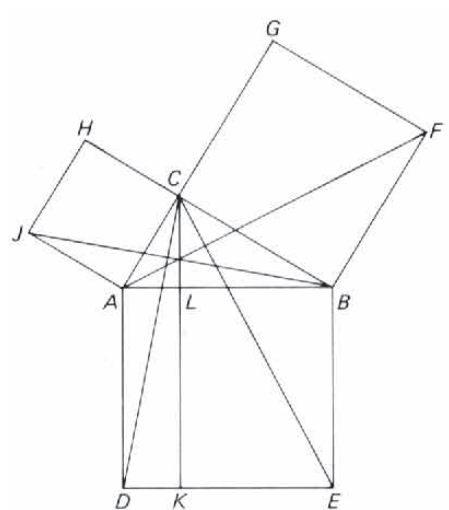

Figure 1: Construction necessary for Euclid's proof of Pythagoras' theorem
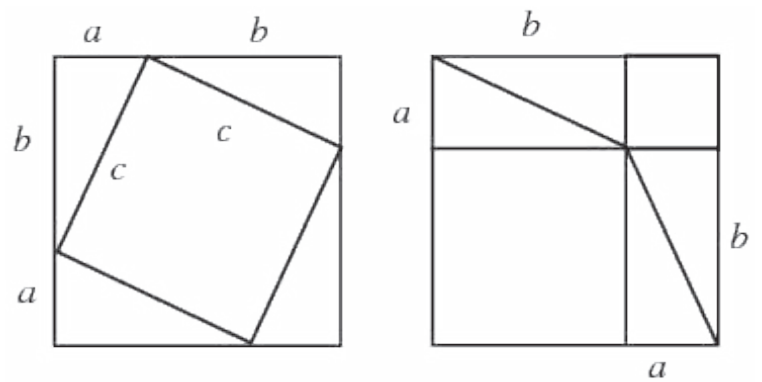

Figure 2: Elegant proof of Pythagoras' theorem

Take four identical right-angled triangles; arrange them as shown in the left half of Figure 2, and draw a frame round them. The area enclosed by the frame is equal to the area occupied by the four triangles plus the area of the central square, which is the square on the hypotenuse. Now rearrange the triangles within the same frame, as shown in the right half of Figure 2. Since the area enclosed by the frame and the area occupied by the four triangles remain the same, it follows that the squares on the hypotenuse must be equal to the sum of the squares on the other side. Now this is worth a "wow" (Glyn, 2010)!

Mathematicians are, in our opinion, more concerned than other scientists about the elegance of their work, and this may be why it seemed natural to start with a pure mathematical example. Nevertheless, although less attention may be given to it, elegance can be found throughout science and engineering, and we want to continue with an example from science and engineering.

An important scientific problem was solved in the 17th century by the British physicist Robert Hooke (Roesler, Harders, Baeker, 2007). He published the solution of his anagram in 1678 as: ut tensio, sic vis ("as the extension, so the force" or "the extension is proportional to the force"). Hooke's law is a principle of physics which states that the force $F$ needed to extend or compress a spring by some distance $X$ is proportional to that distance. That is: $F=-k$ $X$, where $k$ is a constant factor characteristic of the spring, its stiffness. Hooke's law is only a first order linear approximation to the real response of springs and other elastic bodies to applied forces. It must eventually fail once the forces exceed some limit, since no material can be compressed beyond a certain minimum size, or stretched beyond a maximum size, without some permanent deformation or change of state. In fact, many materials will noticeably deviate from Hooke's law well before those elastic limits are reached.

On the other hand, Hooke's law is an accurate approximation for most solid bodies, as long as the forces and deformations are sufficiently small. For this reason, Hooke's law is extensively used in all branches of science and engineering. In its general form, Hooke's law makes it possible to deduce the relation between strain and stress for complex objects in terms of the intrinsic properties of the materials of which it is composed. For example, one can deduce that a homogeneous rod with uniform cross section will behave like a simple spring when stretched, with a stiffness $k$ directly proportional to its cross-section area and inversely proportional to its length. Linear deformations of elastic materials can be approximated as adiabatic. Under these conditions and for quasistatic processes, the first law of thermodynamics for a deformed body can be expressed as

$$
\delta W_{s}=\int_{\partial \Omega} \mathbf{t} \cdot \delta \mathbf{u} \mathrm{dS} ; \delta W_{b}=\int_{\Omega} \mathbf{b} \cdot \delta \mathbf{u} \mathrm{dV}
$$

where $\mathbf{t}$ is the surface traction vector, $\mathbf{b}$ is the body force vector, $\Omega$ represents the body and $\delta \Omega$ represents its surface.

In contrast, we can suppose that materials are isotropic, characterized by properties independent of direction in space. Physical equations involving isotropic materials must therefore be independent of the coordinate system chosen to represent them. The strain tensor is a symmetric tensor. Since the trace of any tensor is independent of 
any coordinate system, the most complete coordinate-free decomposition of a symmetric tensor is to represent it as the sum of a constant tensor and a traceless symmetric tensor. The most general form of Hooke's law for isotropic materials may now be written as a linear combination of two tensors:

$$
\sigma_{i j}=3 K\left(\frac{1}{3} \varepsilon_{k k} \delta_{i j}\right)+2 G\left(\varepsilon_{i j}-\frac{1}{3} \varepsilon_{k k} \delta_{i j}\right) ; \boldsymbol{\sigma}=3 K \operatorname{vol}(\boldsymbol{\varepsilon})+2 G \operatorname{dev}(\boldsymbol{\varepsilon})
$$

where $K$ is the bulk modulus and $G$ is the shear modulus. Are all these expressions elegant? Definitely not. If we want to solve all these equations, we need a great deal of knowledge and time, and it would also be useful to have good technological, computer support.

Using the relationships between the elastic moduli $K$, these equations may also be expressed in various other ways, for example in the simplest way as

$$
\sigma=K \varepsilon
$$

which is definitely the most elegant (and at the same time an adequately accurate expression) for engineering practice and every day (general school) life, because it is unusually effective and simple but at the same time sufficiently accurate. "Wow"! And for the purposes of STEM general education, it is the most appropriate and most economical, thus making it the most elegant solution.

\section{Elegance in Science Education}

What about elegance in Science teaching and learning? Could elegance be defined in the same way? An elegant mathematical proof, an elegant theory, an elegant experiment (or an elegant teaching/learning process) would each be economical, imaginative and sometimes breathtakingly simple, once explained. And that is precisely the point? If we make simplified cost-benefit analyses, we can create a simple equation for use in the educational process:

$$
\text { Used time }+ \text { used effort }+ \text { costs } \frac{\text { must be }}{\cong} \text { quality of knowledge (competence) of the students }
$$

The equation assumes that there are no cause-effect (causal) relationships among the accounted quantities, and that there is only a general relationship. From practice, we know that time, effort and costs affect one another and that there are multiplicative effects between them. From this, we can use the deductive method to conclude that the correct equation should be:

$$
\text { Used time } x \text { used effort } x \text { costs } \frac{\text { must be }}{\cong} \text { quality of knowledge (competence) of the students }
$$

The general trends in education concerning time involve shortening contact hours in school and increasing the amount of independent work students do outside of school (NMC, 2014, Microsoft Partners in Learning, 2010). Concerning effort, teachers need to show increasingly greater effort (students need to be motivated; teachers are required to use methods that are more student friendly - where learning becomes more like play) and the students are becoming "spectators" of the whole process, required to invest less and less effort and responsibility for their own success (Aberšek, Borstner, Bregant, 2014). And concerning costs: with the introduction of modern learning technologies, these costs have drastically increased. Let us take a look at some hypothetical deductible examples (we use normalized (unit) vectors):

- The basic value of students' quality of knowledge is 1.0 . If we decrease time by $20 \%$, the responsibility for success is divided equally between teachers and students, and we increase funding for learning environments by $20 \%$, and take into account the multiplicative effect of individual categories, we get the following value of quality:

$$
0.8(0.5+0.5) 1.2=0.96
$$


- If at the same time we take into account the multiplicative effect of the effort of both participants and the mutual effect, which is equally distributed between the teacher and the student, we get the following:

$$
0.80 .50 .51 .2=0.24
$$

- Teachers need to show more effort (0.7) and students less (0.3) with the same effect of effort (1.0), we get:

$$
0.80 .70 .31 .2=0.202
$$

- If we wish to "increase" the quality by increasing investment: for example, each student gets his own tablet, learning material that is suitably modified, schools are appropriately equipped with wireless connections and other technological solutions, thus hypothetically increasing costs by $30 \%$. If we take into account that we have now additionally burdened the teacher by approximately $10 \%(0.8)$ and unburdened the student of effort and responsibility (to 0.2 ), we get the results for the quality of student knowledge:

$$
0.80 .80 .21 .5=0.192
$$

The numbers are sufficiently self-explanatory. In the end one can only ask the question: Are the trends developing in education "elegant" (economical and imaginative, and sometimes breathtakingly simple once explained), and most importantly are they effective? The answer is within reach with this simple (elegant) mathematical proof.

\section{References}

Aberšek, B., Borstner, B., \& Bregant, J. (2014). The virtual science teacher as a hybrid system: Cognitive science hand in hand with cybernetic pedagogy. Journal of Baltic Science Education, 13 (1), 75-90.

Glynn, I. (2010). Elegance in science, the beauty of simplicity. Oxford: Oxford University Press.

NMC (2014). The Horizon Report Europe: 2014 Schools Edition examines trends, challenges, and technologies for potential impact on and use in teaching, learning, and creative inquiry. Paris: European Commission/The New Media Consortium.

Microsoft Partners in Learning (2010). Bringing a 1-to-1 Program to Life, a Handbook for Senior Secondary School Teachers. New York: Microsoft Corporation.

Roesler, J., Harders, H., Baeker, M. (2007). Mechanical behaviour of engineering materials: Metals, ceramics, polymers, and composites. Berlin: Springer Science \& Business Media.

Boris Aberšek 